\title{
La práctica clínica enfermera en el abordaje de la violencia de género
}

\section{Clinical nurse practice approach to gender violence Enfermeria prática clínica no tratamento de género da violencia}

\author{
Carmen Pérez García ${ }^{1}$, María de los Ángeles Manzano Felipe ${ }^{2}$ \\ ${ }^{1}$ Enfermera Especialista en Salud Mental y en Geriatría \\ ${ }^{2}$ Enfermera Especialista en el Trabajo \\ abordaje de la violencia de género. Cultura de los Cuidados. (Edición digital) 18, 40. \\ Disponible en: http://dx.doi.org/10.7184/cuid.2014.40.14 \\ Correspondencia: Calle María Auxiliadora $n^{\circ} 20$ Portal B $3^{\circ}$ A CP 41008 Sevilla (Sevilla) \\ Correo electrónico: enfermerap8@hotmail.com \\ Recibido: 13/07/2014; Aceptado: 11/10/2014
}

Cómo citar este artículo en edición digital: Pérez García, C; y Manzano Felipe, Ma A. (2014) La práctica clínica enfermera en el

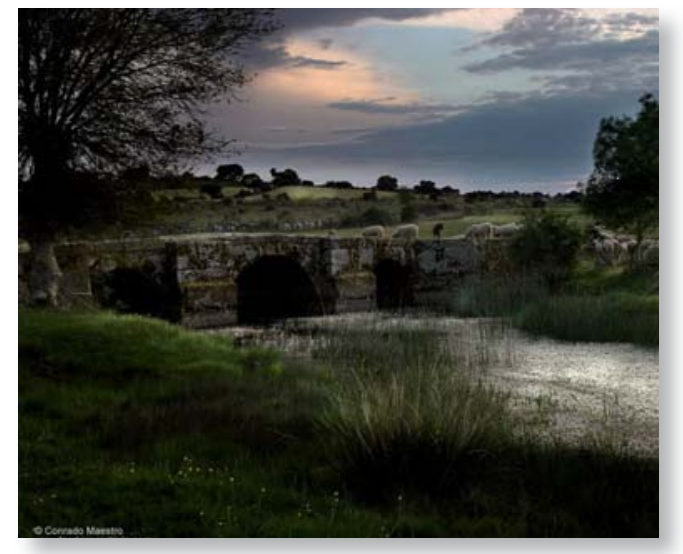

ABSTRACT

Violence against women is a phenomenon that occurs in all countries, social classes and sectors of society. According to the UN definition, gender-based violence (GBV) is "any act or intention that causes harm or physical, sexual or psychological suffering to women, including threats of such acts, coercion or arbitrary deprivation of liberty, and whether in public or private life "the specific needs of these women are multidimensional, so health interventions that take into account the biological, psychological and social aspects are required. This requires the active involvement of all staff in health services is required from a model of comprehensive care. The study aims to determine the basis of nurse for addressing GBV action. The methodology used for this study is qualitative-descriptive research begins with a literature search and continues with the hermeneutic analysis of the documents found. The findings of this study relate to the training of nurses in addressing gender-based violence, as well as the strengths and weaknesses in this regard.

Keywords: Gender violence, Nurse, Enfermera, Clinical Practice, Approach.

\section{RESUMO}

Violência contra a mulher é um fenômeno que ocorre em todos os países, classes sociais e setores da sociedade. De acordo com a definição da ONU, a violência baseada no género (VBG) é "qualquer ato ou intenção que causa dano ou física, sofrimento sexual ou psicológico à mulher, inclusive ameaças de tais atos, coerção ou privação arbitrária de liberdade, e seja na vida pública ou privada "as necessidades específicas dessas mulheres são, por assim intervenções multidimensionais de saúde que levem em conta os aspectos biológicos, psicológicos e sociais são necessários. Isso requer a 
participação activa de todos os funcionários em serviços de saúde é exigido de um modelo de atenção integral. O estudo tem como objetivo determinar a base de enfermeira para tratar de ação VBG. A metodologia utilizada para este estudo é uma pesquisa qualitativa-descritiva começa com uma pesquisa bibliográfica e continua com a análise hermenêutica dos documentos encontrados. As conclusões deste estudo referem-se à formação de enfermeiros no enfrentamento da violência de gênero, bem como os pontos fortes e fracos a este respeito.

Palavras-Chave: Violência de Género, Enfermeira, Prática Clínica, Aproximação.

\section{RESUMEN}

La violencia contra las mujeres es un fenómeno que ocurre en todos los países, clases sociales y ámbitos de la sociedad. Según la definición de la ONU, la violencia de género (VG) es "cualquier acto o intención que origina daño o sufrimiento físico, sexual o psicológico a las mujeres, incluyendo las amenazas de dichos actos, la coerción o privación arbitraria de libertad, ya sea en la vida pública o privada" Las necesidades específicas de estas mujeres son multidimensionales, por lo que se requieren intervenciones sanitarias que tengan en cuenta los aspectos biológicos, psicológicos y sociales. Para ello es necesaria la implicación activa de todo el personal de los servicios sanitarios desde un modelo de atención integral. El estudio tiene como objetivo conocer la base de actuación enfermera para el abordaje de la violencia de género. La metodología usada para este estudio es de investigación cualitativo-descriptiva que se inicia con una búsqueda bibliográfica y continúa con el análisis hermenéutico de los documentos encontrados. Las conclusiones de este estudio versan sobre la formación de las enfermeras en el abordaje de la violencia de género, así como los puntos fuertes y debilidades al respecto.

Palabras Clave: Violencia de Género, Enfermera, Práctica Clínica, Abordaje.

\section{INTRODUCCIÓN}

La violencia de género (VG) es un problema social debido a la discriminación, la desigualdad y las relaciones de poder que establecen los hombres sobre las mujeres (Mendez, 2003) Este tipo de violencia tiene consecuencias graves para la salud y la vida de las mujeres e impone costos económicos considerables, tanto en la familia como en los Servicios de Salud. Se estima que la VG llega a restar uno de cada cinco días de vida saludable de la mujer, lo que se traduce en una menor calidad de vida (Krug 2003) (Olaiz, 2003)

La violencia contra las mujeres es un fenómeno que ocurre en todos los países, clases sociales y ámbitos de la sociedad. Según la definición de la ONU, la violencia de género (VG) es "cualquier acto o intención que origina daño o sufrimiento físico, sexual o psicológico a las mujeres, incluyendo las amenazas de dichos actos, la coerción o privación arbitraria de libertad, ya sea en la vida pública o privada" (ONU, 1993).

Se estima que a nivel mundial aproximadamente el 35\% de las mujeres han sido víctimas de violencia física y/o sexual (OMS, 2013). El porcentaje de mujeres maltratadas que denuncian en España es relativamente bajo, estimándose entre un 2 y $10 \%$ de los casos totales.Según datos del Observatorio contra la Violencia Doméstica y de Género con sede en el Consejo General del Poder Judicial (CGPJ), el número de denuncias de violencia de género registradas en el primer trimestre de 2013 alcanzó los 29.487 casos, con una media de 327 denun- 
cias diarias. Esta cifra implica unas disminución del 5,1\% respecto del cuarto trimestre de 2012, cuando el número de denuncias llegó a las 31.064 (Observatorio contra la Violencia, 2013).

La violencia es un problema de salud pública como lo reconoce la OMS, al señalar que a nivel mundial, cada año se estima que de 10 a $69 \%$ de las mujeres han sufrido maltrato psicológico, físico y sexual, por parte de su compañero Se calcula que cada año mueren en el mundo cuatro millones de mujeres víctimas de la violencia (Krug, 2003)

El objetivo de esta revisión es dar a conocer el abordaje enfermero en la violencia de género, sus características y formación de los profesionales al respecto.

\section{METODOLOGÍA}

La estrategia de búsqueda bibliográfica empelada en este estudio hace uso casi exclusivo de los recursos de internet. La búsqueda se hace principalmente en inglés con los descriptores "Gender violence" "Nurse" "Approach" "Clinical Practice". Se revisan publicaciones desde el 2003 hasta la actualidad. Las fuentes de información consultadas son las siguientes:

\section{Bases de Datos:}

- Biblioteca virtual del Sistema Sanitario Público de Andalucía.

- MEDLINE (www.ncbi.nlm.nih.gov/Pubmed)

- Web of Knowledge (http://www.accesowok.fecyt.es/)

\section{Buscadores académicos:}

-Dialnet (http://dialnet.unirioja.es)

Como se ha encontrado una gran cantidad de documentos relevantes en relación con la violencia de género y su abordaje, nos hemos centrado en aquellos artículos que tratan sobre la importancia del abordaje multidisciplinar, haciendo hincapié en los profesionales de enfermería; formación de los profesionales y uso de los servicios de salud de las personas víctimas de violencia de género.

\section{RESULTADOS}

La revisión bibliográfica realizada se fundamenta en los resultados sobre el abordaje de la violencia de género en los servicios de salud.

\section{Marco legal}

La Ley Orgánica 1/2004 de Medidas de Protección Integral contra la Violencia de Género estableció la creación de una Comisión contra la violencia de género, destinada a prestar apoyo técnico, orientar la planificación de medidas sanitarias y proponer actuaciones para la aplicación de un protocolo sanitario, entre otras competencias (Ley Orgánica 2004).

Además la Ley establece que las Administraciones Sanitarias, en el seno del Consejo Interterritorial del SNS, promoverán las actuaciones de los profesionales sanitarios que permitan la detección precoz de la violencia de género; propondrán el desarrollo de programas de sensibilización y formación continuada del personal sanitario que permitan impulsar el diagnóstico precoz, la asistencia y la rehabilitación de la mujer maltratada (artículo 15); y promoverán la aplicación, la puesta al día, y la difusión de protocolos que contengan pautas homogéneas de actuación ante este problema (artículo 32.3).

Por otra parte, el diagnóstico y la atención a la violencia de género, tanto en el ámbito de la asistencia primaria como en el de la especializada, están incluidos en la cartera de servicios comunes del SNS (Real Decreto 
1030/2006). Por tanto, existe un reciente desarrollo normativo e institucional que asigna responsabilidades al sistema sanitario en la detección y atención sanitaria de la violencia de género (VG), así como en la formación del personal sanitario y en el desarrollo de instrumentos específicos (protocolos).

Desde el año 2007 existe un "Protocolo común para la actuación sanitaria ante la violencia de género". Se trata de un instrumento elaborado de forma colectiva por el Observatorio de Salud de la Mujer, de la Dirección General de la Agencia de Calidad del Sistema Nacional de Salud del Ministerio de Sanidad y Consumo y por la Comisión contra la Violencia de Género, del Consejo Interterritorial del Sistema Nacional de Salud; es decir, un texto consensuado por representantes de la administración central y las autonómicas. Por tanto, debería ser considerado el "suelo mínimo" a partir del cual se desarrollaran las correspondientes políticas en cada uno de los territorios autonómicos; la presentación de la ministra de Sanidad afirma que el texto "se propone para su aplicación en el conjunto del SNS” (Servicio Andaluz de Salud 2008).

\section{Origen y factores de riesgo}

En la actualidad, la violencia contra las mujeres en España sigue siendo todavía un fenómeno "invisibilizado" e inmerso en el ámbito privado en la mayoría de las situaciones. La violencia contra las mujeres tiene hondas raíces sociales y culturales y está vinculada al desequilibrio en las relaciones de poder entre hombres y mujeres en los ámbitos social, económico, religioso y político, pese a los indudables avances en las legislaciones nacionales e internacionales a favor de la igualdad de derechos (Blanco, 2004) (Rangel da Silva, 2011)

De acuerdo con los resultados de algunos estudios, las mujeres en situaciones de mayor riesgo son aquellas que han sido testigos o víctimas de violencia en su infancia, que sufren o han sufrido aislamiento social, que son dependientes económicamente y poseen un bajo nivel educativo (Ruiz-Perez, 2006) (Vives, 2009) (Zorrilla, 2009) (Montero, 2012) . Especialmente mujeres con gran interiorización de valores tradicionalmente "femeninos" como son la sumisión y la obediencia; mujeres que no han desarrollado proyectos de vida propios y cuya vida está en función de los demás (Mazarrasa, 2011).

El factor principal de riesgo para la violencia contra las mujeres es, precisamente, el hecho de ser mujer.

La violencia contra las mujeres es además instrumental. El poder de los hombres y la subordinación de las mujeres, que es un rasgo básico del patriarcado, requiere de algún mecanismo de sometimiento. En este sentido, la violencia contra las mujeres es el modo de afianzar ese dominio. La violencia de género más que un fin en sí mismo, es un instrumento de dominación y control social. Y en este caso se utiliza como mecanismo de mantenimiento del poder masculino y de reproducción del sometimiento femenino. Los hombres maltratadores han aprendido a través del proceso de socialización -que es diferente para mujeres y hombres- que la violencia es la mejor forma de conseguir el control y dominar a la mujer.

\section{Tipos de maltrato}

En la actualidad se definen diferentes formas de violencia contra las mujeres:

\section{- Violencia física}

Comprende cualquier acto no accidental que implique el uso deliberado de la fuerza, como bofetadas, golpes, palizas, empujones, heridas, fracturas o quemaduras, que provoquen o puedan provocar una lesión, daño o 
dolor en el cuerpo de la mujer. Es importante no olvidar que cualquier forma de violencia física es también una violencia psicológica.

\section{- Violencia sexual}

Ocurre siempre que se impone a la mujer, mediante el chantaje, las amenazas o la fuerza, un comportamiento sexual contra su voluntad, se produzca por parte de su pareja o por otras personas.

Existen diferentes manifestaciones de violencia sexual:

\section{- Violencia sexual que no implica contacto} corporal

Exhibicionismo, forzar a ver material pornográfico, mensajes obscenos por correo electrónico o telefónicos, gestos y palabras obscenos, insultos sexistas, acoso sexual, proposiciones sexuales indeseadas, voyeurismo.

\section{- Violencia sexual con contacto corporal}

- Tocamientos, la imposición de relaciones sexuales o prácticas no deseadas, obligar a adoptar posturas que la mujer considera degradantes.

- Violación.

\section{- Violencia contra los derechos sexuales y re- productivos de las mujeres}

Incluye cualquier actuación que restrinja a las mujeres el ejercicio de su derecho a la salud sexual y reproductiva, afectando su libertad para disfrutar de una vida sexual sin riesgos para su salud, así como ejercer libremente su derecho a la maternidad.

\section{- Violencia psicológica}

Conducta intencionada y prolongada en el tiempo, que atenta contra la integridad psíquica y emocional de la mujer y contra su dignidad como persona, y que tiene como objetivo

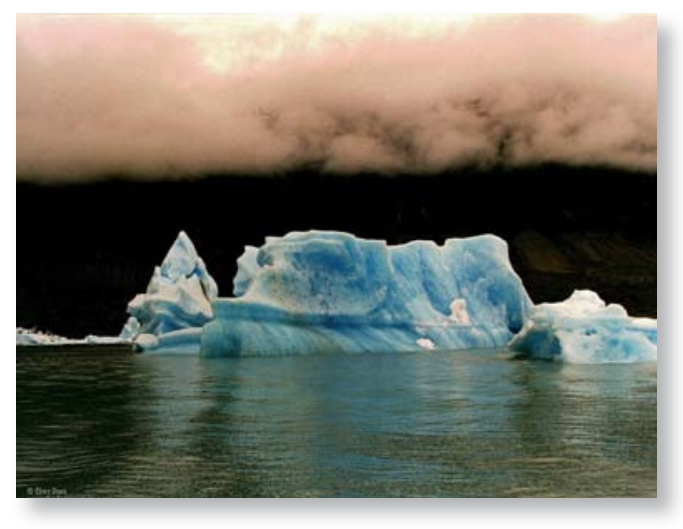

imponer las pautas de comportamiento que el hombre considera que debe tener su pareja. Sus manifestaciones son: las amenazas, insultos, humillaciones o vejaciones, la exigencia de obediencia, el aislamiento social, la culpabilización, la privación de libertad, el control económico (violencia económica), el chantaje emocional, el rechazo o el abandono. Este tipo de violencia no es tan visible como la física o la sexual, es más difícil de demostrar, y en muchas ocasiones no es identificada por la víctima como tal sino como manifestaciones propias del carácter del agresor. Algunas de estas formas de violencia pueden considerarse específicas para determinados grupos vulnerables, como por ejemplo la omisión de cuidados o la medicación negligente en el caso de mujeres con discapacidad, mayores o con enfermedad mental grave.

Además, en el caso de la violencia ejercida contra las mujeres por la pareja o expareja, deben tenerse en cuenta dos elementos importantes: la reiteración de los actos violentos (Aguar-Fernández 2006) y la situación de dominio del agresor, que utiliza la violencia para el sometimiento y control de la víctima.

Es importante tener presente que cualquiera de las formas de violencia de género que se ejerza, tiene consecuencias en todas las dimensiones de la salud: física, psicológica, sexual, reproductiva y social. 


\section{Mitos y falsas creencias}

Se ha argumentado que el consumo de alcohol y otras drogas es la causa de las conductas violentas. Aunque el consumo de alcohol y otras sustancias se asocia con frecuencia a situaciones de violencia, también hay hombres que abusan del alcohol sin que por ello manifiesten un comportamiento violento y muchas agresiones contra las mujeres se ejercen en ausencia de alcohol. Se ha argumentado también que ciertas características personales de las mujeres que padecen violencia de género podrían ser la causa del maltrato.

Por ejemplo, algunas corrientes han recurrido a características de masoquismo o de patologías como la histeria o el trastorno de personalidad dependiente, para explicar por qué algunas mujeres permanecen o regresan a una relación de maltrato.

En la actualidad existe suficiente conocimiento documentado que demuestra que no existen características psíquicas diferentes antes del inicio del maltrato entre las mujeres que lo sufren y las que no, sino que los trastornos y problemas psicológicos de las mujeres maltratadas son una consecuencia del maltrato y no la causa.

\section{¿Cual es el papel del personal sanitario?}

El sistema sanitario también tiene un importante papel en la prevención de la violencia de género, que se articula a través de actuaciones en el ámbito de la sensibilización y formación de profesionales y de atención a la salud integral de las mujeres, lo que incluye actuaciones de salud comunitaria y educación para la salud.

Las necesidades específicas de estas mujeres son multidimensionales, por lo que se requieren intervenciones sanitarias que tengan en cuenta los aspectos biológicos, psicológicos y sociales. Para ello es necesaria la implicación activa de todo el personal de los servicios sanitarios desde un modelo de atención integral.

El modelo de atención integral a la salud incorpora el concepto de INTERDISCIPLINA, entendida como una forma de articulación, que a partir del diálogo y la puesta en común de diversos saberes y habilidades, posibilita la construcción de un nuevo conocimiento así como de una experiencia y un lenguaje que es fruto del abordaje compartido de los conflictos y las situaciones problemáticas que afronta la persona.

La OMS enumera unas recomendaciones básicas para los servicios sanitarios en el abordaje de la violencia de género:

- Preguntar con regularidad, cuando sea factible, a todas las mujeres sobre la existencia de violencia doméstica, como tarea habitual dentro de las actividades preventivas.

- Estar alerta a posibles signos y síntomas de maltrato y hacer su seguimiento.

- Ofrecer atención sanitaria integral y registrarla en la historia de salud o historia clínica.

- Ayudar a entender su malestar y sus problemas de salud como una consecuencia de la violencia y el miedo.

- Informar y remitir a las pacientes a los recursos disponibles de la comunidad.

- Mantener la privacidad y la confidencialidad de la información obtenida.

- Estimular y apoyar a la mujer a lo largo de todo el proceso, respetando su propia evolución.

- Evitar actitudes insolidarias o culpabilizadoras ya que pueden reforzar el aislamiento, minar la confianza en ellas mismas y restar la probabilidad de que busquen ayuda.

- Establecer una coordinación con otros y otras profesionales e instituciones. 
- Colaborar en dimensionar e investigar el problema mediante el registro de casos.

\section{Formación para el abordaje de la violencia de género}

Las actitudes y creencias de los profesionales sanitarios juegan un papel fundamental para el futuro abordaje del problema (Macias, 2012). Un estudio realizado en la Escuela de Ciencias de la Salud de la Universidad de Sevilla analiza la autopercepción, socialización, formación académica en género y la capacitación de los estudiantes de Enfermería como futuros profesionales de la salud. Los participantes manifiestan haber recibido influencia en su formación en género principalmente de los medios de comunicación $(97,7 \%)$ y de la familia $(95,5 \%)$, seguidos en frecuencia por el instituto $(88,3 \%)$, la universidad $(87,5 \%)$ y la escuela $(76,2 \%)$. Mayoritariamente $(82,6 \%)$ reconocen haber trabajado contenidos de género en asignaturas de la universidad y afirmar que/ la formación para detectar los tipos de violencia es básica. Consideran que su capacitación para detectar la violencia, según los distintos tipos, es: física el 95,5\%, psicológica $88,7 \%$, abuso sexual 73,6\% y otras formas el 28,3\%.

En este estudio, además, y coincidiendo con los resultados de trabajos anteriores, se destaca las diferencias existentes en la percepción global del fenómeno entre mujeres y hombres, siendo éstos últimos quienes muestran una actitud más sexista, respondiendo así a la presión social que marcan los estereotipos masculinos. Estas creencias disminuyen tanto en hombres como en mujeres a medida que aumenta su formación específica en el tema.

Por tanto, es evidente la importancia de la formación profesional para la detección, seguimiento y apoyo en la toma de decisiones. Esta necesidad continua de capacitación efectiva de los profesionales de la salud para abordar la violencia de pareja contra las mujeres se ha puesto de manifiesto en numerosas ocasiones por organismos internacionales, así como por las propias organizaciones profesionales (Consejo General de Enfermería, 2013).

El nivel de conocimientos puede valorarse como medio-alto en trabajadores de AP, lo que refleja el esfuerzo realizado en los últimos años y la toma de conciencia por parte de los profesionales aunque se siguen explicitando barreras organizativas importantes (Arredondo, 2012).

\section{Abordaje enfermero}

El Consejo General de Enfermería español destaca que existen principalmente dos campos en el abordaje de la Violencia de Género: el educativo y el sanitario, sobre todo en el ámbito de la prevención, detección y rehabilitación de este problema (Consejo General de Enfermería 2013). La formación debería incluir el aprendizaje de la entrevista psicosocial $\mathrm{y}$, fundamentalmente, la adquisición de ciertas habilidades, como la acogida, la escucha y la contención.

En el ámbito sanitario se están desarrollando programas formativos encaminados a potenciar las habilidades de las enfermeras en detectar, informar y evaluar los malos tratos. Estudios realizados con personal de Enfermería demuestran que la tasa autocomunicada de maltrato como causa de lesiones puede mejorar de un 7,5\% hasta un $30 \%$ cuando la mujer es atendida por enfermeras formadas en la materia (De la Fuente, 2005).

Los profesionales sanitarios, especialmente las enfermeras, constituyen un grupo fundamental, no sólo en la atención, sino en la prevención, detección precoz e identificación de la violencia de género. Los profesionales de Enfermería intervienen en la detección de signos y en la recepción y acogida de las vícti- 


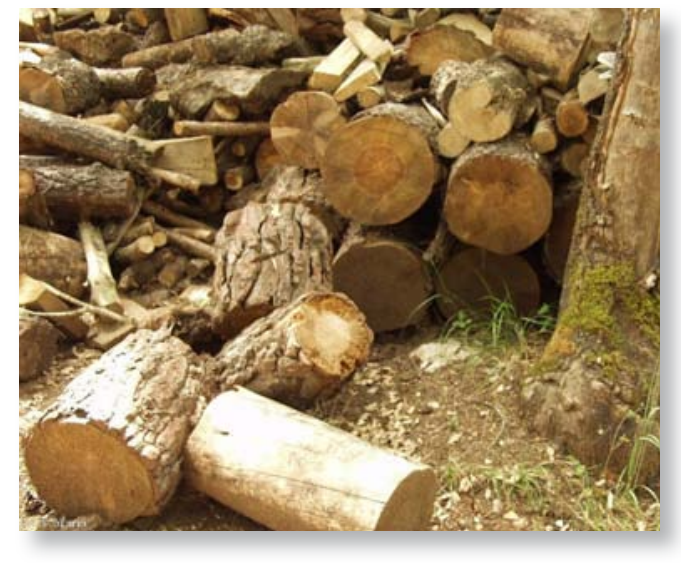

mas, siendo una pieza clave en el seguimiento del proceso, en el asesoramiento y apoyo psicológico, en la garantía de la continuidad de cuidados y como persona referente, cercana y accesible a la mujer durante todo el proceso (Vargas, 2006) (Crespo, 2006). La formación de los profesionales enfermeros en el conocimiento de las medidas de protección instauradas así como el trabajo en equipo es fundamental para hacer frente a este problema. Es importante reconocer la labor enfermera también en la elaboración de un plan de cuidados integrado e individualizado y de la educación de la sociedad.

\section{CONCLUSIONES}

Es evidente que la violencia de género constituye un grave problema de salud pública que requiere de un abordaje integral e integrado desde una perspectiva interdisciplinar. Los profesionales de Enfermería tienen un papel fundamental en la atención a las mujeres víctimas de este problema, siendo una responsabilidad ética y profesional diseñar, implementar y evaluar programas que respondan a las necesidades específicas de este colectivo. Los centros de salud, los servicios de planificación familiar, salud mental y urgencias pueden ser servicios diana para la prevención, la detección precoz y la atención de la mujer maltratada.
La formación y una adecuada gestión de los recursos existentes son dos de los elementos claves en este proceso. Además, sería importante contemplar la coordinación con los sectores educativos, los servicios sociales y los servicios jurídicos y policiales, así como la participación de las asociaciones de mujeres.

\section{BIBLIOGRAFÍA}

- Aguar-Fernandez, M; Delgado-Sanchez, A; CastellanoArroyo, M. (2006) Luna del Castillo, JD. Prevalencia de malos tratos en mujeres que consultan al médico de familia. Aten Primaria 37:241-2.

- Arredondo-Provecho, A.B; Broco-Barredo, M; AlcaláPonce de León, T; Rivera-Álvarez, A; Jiménez Trujillo, I; Gallardo-Pino, C. (2012) Profesionales de atención primaria de Madrid y violencia de pareja hacia la mujer en el año 2012. Rev Esp Salud Publica;86(1):85-99.

- Blanco, P; Ruíz Jarabo, C; García de Vinuesa, L; Martín García, M. (2004) La violencia de pareja y salud de las mujeres. Gaceta Sanitaria 18 (1): 182-188.

- Consejo General de Enfermería. Posicionamiento del Consejo General de Enfermería ante la Violencia de Género. Disponible en: http://www.ecs.enfermundi. com/fileadmin/Documentos_de_la_profesion/posicionamiento_Violencia_genero.pdf Downloaded: 11 th December 2013

- Crespo, M; Arinero, M. (2010) Assessment of the efficacy of a psychological treatment for women victims of violence by their intimate male partner. Span J Psychol. 13(2):849-63.

- De la Fuente Aparicio. D; Salamanca Castro, A.B; Sánchez Castro, S. (2005) ¿Se encuentran capacitadas las enfermeras para detectar malos tratos en Atención Primaria? Nure Investigación 15:1-10.

- Krug G.E; Dahlberg, L.L; Mercy, J.A; Zwi, A.B; Lozano, R. (2003) Informe mundial sobre la violencia y la salud. EUA: OMS-OPS: 97.

- Ley orgánica 1/2004, de 28 de diciembre, de medidas de protección integral contra la violencia de género. BOE de 28 de diciembre de 2004. Disponible: http://www.boe. es/boe/dias/2004/12/29/pdfs/A42166-42197.pdf] 
- Macías Seda, J; Gil García, E; Rodríguez Gázquez, Mª.A; González López, J.R; González Rodríguez, MªM. Soler Castells, A.M. (2012) Creencias y actitudes del alumnado de Enfermería sobre la violencia de género. Index de Enfermería 21 (1-2): 1-12.

- Mazarrasa Alvear, L; Díaz Rodríguez, D.R. (2011) Violencia de género y cuidados de Enfermería. Metas de Enfermería 41: 52- 57.

- Méndez-Hernández, P. Valdez-Santiago, R. Viniegra-Velásquez, L. Rivera-Rivera, L. Salmerón-Castro, J (2003) Violencia contra la mujer: conocimiento y actitud del personal médico del Instituto Mexicano del Seguro Social, Morelos, México. Sal Pub Mex 45: 472-82.

- Montero, I; Ruiz-Pérez, I; Escribà-Agüir, V; Vives-Cases, C; Plazaola-Castaño, J; Talavera M; Martín-Baena, D; Peiró, R. (2012) Strategic responses to intimate partner violence against women in Spain: a national study in primary care. J. Epidemiol Community Health 66(4):352.

- Observatorio contra la Violencia (2013) Estadísticas sobre la violencia de género. Fundación Mujeres. Disponible en: http://www.observatorioviolencia.org/documentos.php?id=316. Downloaded: 26th April 2014

- Olaiz,G; Rico, B; Del Río, A. (2003) Encuesta Nacional sobre Violencia contra las Mujeres.. Instituto Nacional de Salud Pública, Cuernavaca Mor

- OMS (2013) Estimaciones mundiales y regionales de la violencia contra la mujer: prevalencia y efectos de la violencia conyugal y de la violencia sexual no conyugal en la salud;. Available http://apps.who.int/iris/bitstream/10665/85243/1/WHO_RHR_HRP_13.06_spa. pdf. Downloaded: 22th May 2014

- Organización de las Naciones Unidas. Declaración sobre la eliminación de la violencia contra la mujer de 1993 (48/ 104). Disponible en: http://www.acnur.org/biblioteca/pdf/1286.pdf [Acceso 02/05/2014]

- Rangel da Silva, L; Domingues Bernardes Silva, M; Mota Xavier de Meneses, T; Rodríguez Borrego, M.A; Meneses dos Santos, I.M; Lemos, A. (2011) El fenómeno de la violencia de género en la mujer a partir de la producción científica de Enfermería. Rev. Enfermería Global 22:1-11

- Ruiz-Pérez, I; Plazaola-Castaño, J; Alvarez-Kindelán,M; Palomo-Pinto, M; Arnalte-Barrera, M; Bonet-Pla, A; De
Santiago-Hernando, M.L; Herranz-Torrubiano, A; Garralón-Ruiz, L.M. (2006) Sociodemographic associations of physical, emotional, and sexual intimate partner violence in Spanish women. Ann Epidemiol16(5):357-63.

- Servicio Andaluz de Salud. Consejería de Salud. Junta de Andalucía (2008) Protocolo andaluz para la actuación sanitaria ante la violencia de género.

- Vargas Matute, A; Dorado, M.I; Alonso, M.J; Díaz, M; Blasco, E.J. (2006) Importancia del papel de la Enfermería de urgencias en la detección de violencia de género. Rev. Presencia 2 (3). Disponible en <http://www.index-f. com/presencia/n3/35articulo.php $>$

- Vives-Cases, C; Alvarez-Dardet, C; Gil-González, D; Torrubiano-Domínguez, J; Rohlfs, I; Escribà-Agüir, V. (2009) Perfil sociodemográfico de las mujeres afectadas por violencia del compañero íntimo en España. Gac Sanit 23(5):410-4.

- Zorrilla, B; Pires, M; Lasheras, L; Morant, C; Seoane, L; Sanchez, L.M; Galán, I; Aguirre, R; Ramírez, R; Durbán, M. (2009) Intimate partner violence: last year prevalence and association with socio-economic factors among women in Madrid, Spain. Eur J Public Health 20(2):169-75.

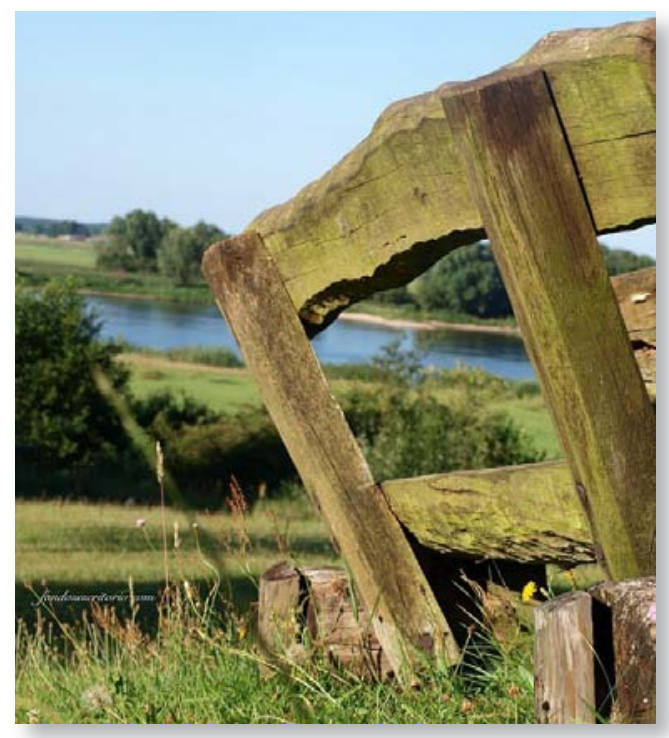

